\title{
Manifestly Lorentz Invariant Chiral Boson Action
}

\author{
Paul K. Townsend (우 \\ Department of Applied Mathematics and Theoretical Physics, Centre for Mathematical Sciences, University of Cambridge, \\ Wilberforce Road, Cambridge CB3 OWA, United Kingdom
}

(Received 17 December 2019; accepted 20 February 2020; published 13 March 2020)

\begin{abstract}
A manifestly Lorentz invariant action is found for the Floreanini-Jackiw chiral boson. The method involves a novel chiral reduction of the phase-space action for a string and can be adapted to describe chiral bosons on the heterotic string worldsheet. A similar manifestly Lorentz invariant action is found for an entire class of conformal chiral $2 k$-form electrodynamics in $(4 k+2)$ dimensions which includes the Floreanini-Jackiw theory as the $k=0$ case.
\end{abstract}

DOI: 10.1103/PhysRevLett.124.101604

Maxwell's conformal-invariant equations for electrodynamics have a natural generalization to $n$-form electrodynamics in a Minkowski spacetime of $2(n+1)$ dimensions, and these equations are derivable from a Lorentz-invariant action quadratic in the gauge-invariant $(n+1)$-form field strength (see e.g., [1]). For $n=2 k$ one may consistently impose a self-duality condition on this $(2 k+1)$-form; the resulting parity-violating, but still conformal invariant, equations are those of "chiral $2 k$-form electrodynamics" (see e.g., [2]). However, the self-duality condition makes it difficult to find a manifestly Lorentz-invariant action [3], which greatly complicates any attempt to include interactions. Many solutions to this problem have been proposed, each with some feature that could be considered undesirable; the most relevant here is that of Pasti et al. [4] and the most recent is that of Sen [5] to whom we defer for references to other proposals.

The focus here will be on a class of chiral $2 k$-form electrodynamics that includes, as the $k=0$ case, the chiral boson theory of Floreanini and Jackiw [6]. This is a conformally invariant free-field theory for a scalar field $\varphi$ in a 2-dimensional Minkowski spacetime. For time-space coordinates $(t, \sigma)$ the Floreanini-Jackiw $(\mathrm{FJ})$ action is

$$
S_{F J}[\varphi]=\int d t \int d \sigma\left(\dot{\varphi}-\varphi^{\prime}\right) \varphi^{\prime},
$$

where the overdot indicates a partial time derivative and a prime indicates a derivative with respect to $\sigma$. The corresponding field equation is

Published by the American Physical Society under the terms of the Creative Commons Attribution 4.0 International license. Further distribution of this work must maintain attribution to the author(s) and the published article's title, journal citation, and DOI. Funded by SCOAP .

$$
\partial_{-} \varphi^{\prime}=0 \quad\left[\partial_{ \pm}=\frac{1}{2}\left(\partial_{t} \pm \partial_{\sigma}\right)\right] .
$$

This is similar to the manifestly Lorentz invariant free chiral boson equation $\partial_{-} \varphi=0$, but with $\varphi^{\prime}$ replacing the scalar field $\varphi$. Neither the FJ action nor its field equation is manifestly Lorentz invariant but the first-order variation of the action under a Lorentz transformation is a surface term [6], and this implies Lorentz invariance of Eq. (2); its general solution is

$$
\varphi=\varphi_{0}(t)+\Phi\left(\xi^{+}\right) \quad\left(\xi^{ \pm}=t \pm \sigma\right),
$$

but the zero mode is unphysical because the FJ action has a (restricted) gauge invariance: $\varphi(\xi) \rightarrow \varphi(\xi)+a(t)$ for arbitrary function $a(t)$.

One purpose of this Letter is to show that the FJ action is a gauge-fixed version of a manifestly Lorentz invariant action constructed from fields that transform linearly under the Lorentz group. This "covariant" action can be interpreted as a "chiral dimensional reduction" of the action for a string in a three-dimensional (3D) spacetime, and the method can be adapted to provide a covariant action for chiral bosons on the heterotic string.

Another purpose of this Letter is to show that the FJ chiral boson theory, in both its original and covariant forms, is the special $k=0$ case of a class of conformal chiral $2 k$-form electrodynamics in a $(4 k+2)$-dimensional Minkowski spacetime. The $k=1$ case and its relation to the M5-brane $[7,8]$ will be briefly reviewed towards the end of this Letter before consideration of $k>1$.

We begin with the 3D string of tension $T$. Let $\left\{X^{m} ; m=\right.$ $0,1,2\}$ be Minkowski spacetime coordinates and let $(t, \sigma)$ now be arbitrary worldsheet coordinates. Prior to chiral reduction, the phase-space action is 


$$
S=\int d t \int d \sigma\left\{\dot{X}^{m} P_{m}-e \mathcal{H}_{\perp}-u \mathcal{H}_{\|}\right\}
$$

where the worldvolume fields $P_{m}$ are canonically conjugate to the maps $X^{m}$ from the worldsheet to spacetime, and $(e, u)$ are Lagrange multipliers for phase-space constraints. The constraint functions are

$$
\begin{aligned}
\mathcal{H}_{\perp} & =\frac{1}{2}\left[\eta^{m n} P_{m} P_{n}+T^{2} \eta_{m n}\left(X^{m}\right)^{\prime}\left(X^{n}\right)^{\prime}\right] \\
\mathcal{H}_{\|} & =\left(X^{m}\right)^{\prime} P_{m} .
\end{aligned}
$$

As is well known, these constraints are first class and their Poisson bracket (PB) algebra is the Lie algebra of the infinite-dimensional 2D conformal group. The gauge transformations generated by the constraints are on-shell equivalent to worldsheet diffeomorphisms.

We shall now break the 3D Lorentz invariance to 2D Lorentz invariance by making the identification $X^{2} \sim X^{2}+$ $2 \pi R$. On setting

$$
X^{2}=R \varphi, \quad R P_{2}=P_{\varphi},
$$

we get a phase-space action of the form

$$
S=\int d t \int d \sigma\left\{\dot{X}^{\mu} P_{\mu}+\dot{\varphi} P_{\varphi}-e \mathcal{H}_{\perp}-u \mathcal{H}_{\|}\right\},
$$

where $\mu=0,1$ and $\varphi \sim \varphi+2 \pi$. We shall suppose (in units for which $\hbar=1$ ) that

$$
T R^{2}=1,
$$

in which case the constraint functions can be written as

$$
\begin{aligned}
\mathcal{H}_{\perp} & =\frac{1}{2}\left[P^{2}+T\left(P_{\varphi}^{2}+\left(\varphi^{\prime}\right)^{2}\right)+T^{2}\left(X^{\prime}\right)^{2}\right], \\
\mathcal{H}_{\|} & =\left(X^{\mu}\right)^{\prime} P_{\mu}+\varphi^{\prime} P_{\varphi},
\end{aligned}
$$

where $P^{2}=\eta^{\mu \nu} P_{\mu} P_{\nu}$ and $\left(X^{\prime}\right)^{2}=\eta_{\mu \nu}\left(X^{\mu}\right)^{\prime}\left(X^{\nu}\right)^{\prime}$. The surviving $2 \mathrm{D}$ Lorentz group has only one generator, a Lorentz boost, and its Noether charge is

$$
L=\int d \sigma\left(X^{0} P^{1}-X^{1} P^{0}\right) .
$$

To get some intuition into what has been done so far, we may impose the following Monge gauge conditions:

$$
X^{0}=t, \quad X^{1}=\sigma .
$$

In this gauge the constraints can be solved for $P_{\mu}$ :

$$
P_{0}=\mp \sqrt{\left[T+\left(\varphi^{\prime}\right)^{2}\right]\left[T+P_{\varphi}^{2}\right]}, \quad P_{1}=-\varphi^{\prime} P_{\varphi} .
$$

Notice that the energy density $P^{0}$ has no $T \rightarrow \infty$ limit unless we subtract $T$ from it. This may be achieved by making the following replacement in the constraints:

$$
P_{\mu} \rightarrow \mathbb{P}_{\mu}=P_{\mu} \mp T \varepsilon_{\mu \nu}\left(X^{\nu}\right)^{\prime} .
$$

This has no effect on the algebra of constraint functions but in the Monge gauge we now have

$$
\mathbb{P}_{0}=P_{0} \mp T, \quad \mathbb{P}_{1}=P_{1},
$$

where the $P_{\mu}$ are as in (12). For the same sign choice as before we now have the Hamiltonian density

$$
P^{0}=\sqrt{\left[T+\left(\varphi^{\prime}\right)^{2}\right]\left[T+P_{\varphi}^{2}\right]}-T .
$$

Notice that

$$
\lim _{T \rightarrow \infty} P^{0}=\frac{1}{2}\left[P_{\varphi}^{2}+\left(\varphi^{\prime}\right)^{2}\right] .
$$

In this limit, elimination of $P_{\varphi}$ yields the standard Lorentz invariant free-field action for massless scalar field $\varphi$.

To get a chiral boson action from the string action (7) we must include an additional constraint: $P_{2} \equiv T X_{2}^{\prime}$. Given the periodic identification of $X_{2}$ and the relation (8), this constraint is equivalent to

$$
\chi(\sigma) \equiv 0, \quad \chi=P_{\varphi}-\varphi^{\prime} .
$$

Expansion of $\chi(\sigma)$ on a set of basis functions yields one zero mode [generator of the $\varphi \rightarrow \varphi+a(t)$ gauge transformation of the FJ theory] and a set of second-class constraints which would require, for some purposes, a replacement of Poisson brackets by Dirac brackets. This complication can be avoided by simply substituting $\varphi^{\prime}$ for $P_{\varphi}$ in (7) to get the new action

$$
S=\int d t \int d \sigma\left\{\dot{X}^{\mu} P_{\mu}+\dot{\varphi} \varphi^{\prime}-e \mathcal{H}_{\perp}-u \mathcal{H}_{\|}\right\},
$$

where now

$$
\begin{aligned}
\mathcal{H}_{\perp} & =\frac{1}{2}\left[\mathbb{P}^{2}+2 T\left(\varphi^{\prime}\right)^{2}+T^{2}\left(X^{\prime}\right)^{2}\right], \\
\mathcal{H}_{\|} & =\left(X^{\mu}\right)^{\prime} P_{\mu}+\left(\varphi^{\prime}\right)^{2} .
\end{aligned}
$$

We have included here the replacement of (13), which affects only $\mathcal{H}_{\perp}$. The canonical PB relations determined by this action are

$$
\begin{aligned}
\left\{X^{\mu}(\sigma), P_{\nu}(\varsigma)\right\}_{P B} & =\delta_{\nu}^{\mu} \delta(\sigma-\varsigma), \\
\{\varphi(\sigma), \varphi(\varsigma)\}_{P B} & =-\frac{1}{2} \epsilon(\sigma-\varsigma),
\end{aligned}
$$


where $\epsilon^{\prime}(\sigma)=\delta(\sigma)$; the second line is also the Dirac bracket relation required for consistency with the chirality constraint when this is introduced via a Lagrange multiplier field. It is convenient to choose a functional basis for the other constraints by defining

$$
H_{\perp}[\beta]=\int d \sigma \beta \mathcal{H}_{\perp}, \quad H_{\|}[\alpha]=\int d \sigma \alpha \mathcal{H}_{\|},
$$

where $\alpha$ is a 1D vector field and $\beta$ a scalar inverse-density, equivalent to a vector field in $1 \mathrm{D}$. Assuming that $(\alpha, \beta)$ are smooth, and have compact support, one finds that

$$
\begin{aligned}
& \left\{H_{\perp}[\beta], H_{\perp}[\tilde{\beta}]\right\}_{P B}=T^{2} H_{\|} \llbracket \beta, \tilde{\beta} \rrbracket, \\
& \left\{H_{\|}[\alpha], H_{\perp}[\beta]\right\}_{P B}=H_{\perp} \llbracket \alpha, \beta \rrbracket, \\
& \left\{H_{\|}[\alpha], H_{\|}[\tilde{\alpha}]\right\}_{P B}=H_{\|} \llbracket \alpha, \tilde{\alpha} \rrbracket,
\end{aligned}
$$

where $[\cdot, \cdot]$ indicates a commutator of (1D) vector fields. As this is the same result that one finds prior to the introduction of the chirality constraint, we may again impose the Monge gauge conditions (11) and solve the constraints for $P_{\mu}$, but this now yields (for the same sign choice as before) the $T$-independent result

$$
P_{0}=-\left(\varphi^{\prime}\right)^{2}, \quad P_{1}=-\left(\varphi^{\prime}\right)^{2} .
$$

This gives $P^{0}=\left(\varphi^{\prime}\right)^{2}$, and the Monge gauge action becomes the FJ chiral boson action (1).

We have now found a covariant action for the FJ chiral boson equation but, as things stand, this has been accomplished at the cost of introducing the dimensionful constant $T$. Moreover, this $T$ dependence cannot be removed from the covariant action by taking $T \rightarrow \infty$ because the PB algebra of its constraint functions is singular in this limit. However, one can take the $T \rightarrow 0$ limit; in this case

$$
\mathcal{H}_{\perp}=\frac{1}{2} P^{2}, \quad \mathcal{H}_{\|}=\left(X^{\mu}\right)^{\prime} P_{\mu}+\left(\varphi^{\prime}\right)^{2} .
$$

Apart from the fact that the string worldsheet fills the 2D spacetime, implying an absence of any dynamics if $\varphi^{\prime} \equiv 0$ the constraints are those of the null, or tensionless, string [9] modified by the $\left(\varphi^{\prime}\right)^{2}$ term in $\mathcal{H}_{\|}$. This suggests a nullstring dust [10] interpretation, and hence conformal invariance, which is readily verified: the corresponding Noether charges are

$$
Q_{(k)}=-\int d \sigma k^{\mu}(X) P_{\mu}
$$

for any 2D conformal Killing vector field $k(X)$.

A covariant action for the FJ chiral boson has now been found via the introduction of additional fields that can be removed by gauge fixing, as for the PST method [4] but here the Lorentz group acts only on the additional fields. Moreover, it acts as an "internal" symmetry that becomes a 2D spacetime Lorentz symmetry only after gauge fixing; this happens because the Monge gauge condition is Lorentz invariant only if a Lorentz transformation is combined with a "compensating" worldsheet diffeomorphism. As the Lorentz boost Noether charge of (10) is diffeomorphism invariant, we may find the Lorentz boost Noether charge of the Monge-gauge action by substitution using (11) and (23):

$$
L \rightarrow L[\varphi]:=-\int d \sigma \xi^{+}\left(\varphi^{\prime}\right)^{2} \quad\left(\xi^{ \pm}=t \pm \sigma\right) .
$$

The Lorentz boost transformation of any function $f$ of phase-space fields is given by the formula

$$
\delta_{w} f=w\{f, L\}_{P B},
$$

where $w$ is the boost parameter. Prior to gauge fixing, $\varphi$ is inert, but after gauge fixing we get the result of [6]:

$$
\delta_{w} \varphi=-w \xi^{+} \varphi^{\prime} \quad \text { (Monge gauge) }
$$

More generally, the Monge-gauge expression for the Noether charge (25) is

$$
Q_{(k)}=\int d \sigma k^{+}(\xi)\left(\varphi^{\prime}\right)^{2} \quad\left(k^{ \pm}=k^{0} \pm k^{1}\right),
$$

which generates the transformation $\delta_{k} \varphi=k^{+} \varphi^{\prime}$. Ignoring surface terms, it may be verified directly that this induces a variation of the FJ action that is a surface term if, and only if, $\partial_{-} k^{+}=0$, as required for a conformal transformation. Using (3) we have $\delta_{k} \Phi=k^{+} \partial_{+} \Phi \equiv \mathcal{L}_{k} \Phi$, which is the first-order variation of a $2 \mathrm{D}$ scalar field under a conformal transformation.

Although the simplest set of constraint functions for the covariant chiral boson action (18) are those of (24), we are free to choose those of (19) and this freedom allows a string-theory application. So far we have considered a chiral reduction on $S^{1}$ of a 3D string. Consider now the chiral reduction on $T^{16}$ of a $26 \mathrm{D}$ string; the result is, for 10D Minkowski coordinates $\left\{X^{M} ; M=0, \ldots, 9\right\}$,

$S=\int d t \int d \sigma\left\{\dot{X}^{M} P_{M}+\dot{\boldsymbol{\varphi}} \cdot \boldsymbol{\varphi}^{\prime}-e \mathcal{H}_{\perp}-u \mathcal{H}_{\|}\right\}$,

where $\varphi$ is a Euclidean 16-vector of FJ scalar fields, and the constraint functions are

$$
\begin{aligned}
\mathcal{H}_{\perp} & =\frac{1}{2}\left[\eta^{M N} P_{M} P_{N}+2 T\left|\boldsymbol{\varphi}^{\prime}\right|^{2}+T^{2} \eta_{M N}\left(X^{M}\right)^{\prime}\left(X^{N}\right)^{\prime}\right], \\
\mathcal{H}_{\|} & =\left(X^{M}\right)^{\prime} P_{N}+\left|\boldsymbol{\varphi}^{\prime}\right|^{2} .
\end{aligned}
$$


In the conformal gauge, $u=0$ and $e=1 / T$, elimination of $P_{M}$ yields the quadratic Lagrangian density

$$
\mathcal{L}=-2 T \partial_{-} X^{M} \partial_{+} X^{N} \eta_{M N}+\left(\dot{\boldsymbol{\varphi}}-\boldsymbol{\varphi}^{\prime}\right) \cdot \boldsymbol{\varphi}^{\prime} .
$$

By adding the 10-vector of antichiral fermions required for $(1,0)$ supersymmetry, and then the conformal-gauge ghosts, we arrive at an anomaly-free conformal-gauge action for the heterotic string in which the center of mass motion is in the 10D Minkowski spacetime because the 16 FJ chiral bosons have no zero modes.

We turn now to the higher-dimensional chiral generalizations of the FJ theory. We start from a $(4 k+2)$ dimensional Minkowski spacetime with Minkowski coordinates $\left(t, \boldsymbol{\sigma}=\left\{\sigma^{i} ; i=1, \ldots, 4 k+1\right\}\right)$. We then introduce a $2 k$-form potential $A(t)$ on the constant-time hypersurfaces, from which we form the gauge-invariant antisymmetric tensor density with components

$$
B^{i_{1} \ldots i_{2 k}}=\frac{1}{(2 k) !} \varepsilon^{i_{1} \cdots i_{2 k} j \ell_{1} \cdots \ell_{2 k}} \partial_{j} A_{\ell_{1} \cdots \ell_{2 k}} .
$$

Next, we propose an action of the form

$$
S[A]=\int d t \int d \boldsymbol{\sigma}\left\{\frac{1}{(2 k) !} \dot{A}_{i_{1} \cdots i_{2 k}} B^{i_{1} \cdots i_{2 k}}-\mathcal{H}\right\},
$$

where $\mathcal{H}$ is some gauge-invariant and rotationally invariant Hamiltonian density; for a "chiral" electrodynamics we expect it to be a function of $B$ only. Consider the choice

$$
\mathcal{H}=\sqrt{T^{2}+2 T|B|^{2}+|B \times B|^{2}}-T,
$$

where $T$ is a constant [with dimensions of $(4 k+1)$-brane tension]. We use the notation

$$
(B \times B)_{i}:=\frac{1}{[(2 k) !]^{2}} \varepsilon_{i j_{1} \cdots j_{2 k} \ell_{1} \cdots \ell_{2 k}} B^{j_{1} \cdots j_{2 k}} B^{\ell_{1} \cdots \ell_{2 k}},
$$

and $|\cdot|$ indicates the Euclidean norm:

$$
\begin{aligned}
|B|^{2} & =\frac{1}{(2 k) !} \delta_{i_{i} j_{1}} \cdots \delta_{i_{2 k} j_{2 k}} B^{i_{1} \cdots i_{2 k}} B^{j_{1} \cdots j_{2 k}}, \\
|B \times B| & =\delta^{i j}(B \times B)_{i}(B \times B)_{j} .
\end{aligned}
$$

The $k=0$ case of (34) is the FJ action, irrespective of the value of $T$. To see this write $A=\varphi$ for the 0 -form potential, so that $B=\varphi^{\prime}$; then $\mathcal{H}=\left(\varphi^{\prime}\right)^{2}$.

For $k \geq 1$ the Hamiltonian density (35) is $T$ dependent. In the $T \rightarrow \infty$ limit it is quadratic in $B$ and the action is the Henneaux-Teitelboim action for free chiral $2 k$-form electrodynamics [2]. For finite $T$ there are interactions but these simplify in the $T \rightarrow 0$ limit:

$$
\left.\mathcal{H}\right|_{T=0}=|B \times B| .
$$

This is homogeneous of degree 2 in $B$ but not quadratic, so it defines a (conformally invariant) interacting theory. The $k=1$ case was found by Gibbons and West [7] from the M5-brane phase-space action of [11] by a limit analogous to that introduced by Bialynicki-Birula in the context of Born-Infeld electrodynamics [12]. It was recently rediscovered by the author [8], and interpreted as an infrared limit of the chiral 2-form electrodynamics on a static planar M5-brane in the $\mathrm{AdS}_{7} \times S^{4}$ vacuum of M-theory; this was inspired by a similar interpretation of Bialynicki-Birula's nonlinear conformal electrodynamics as an infrared limit of the Born-Infeld electrodynamics on a D3-brane in $\mathrm{AdS}_{5} \times S^{5}[13]$.

As we have seen here for $k=0$, and as shown for $k=1$ in [8], the action (34) is Lorentz invariant for the Hamiltonian density of (35) because it is the gauge-fixed version of a reparametrization invariant action that is manifestly Lorentz invariant. We now investigate whether this remains true for $k>1$. The first step is to consider an action of the form

$$
\begin{aligned}
S= & \int d t \int d \boldsymbol{\sigma}\left\{\dot{X}^{\mu} P_{\mu}-\frac{1}{(2 k) !} \dot{A}_{i_{1} \cdots i_{2 k}} B^{i_{1} \cdots i_{2 k}}\right. \\
& \left.-e \mathcal{H}_{\perp}-u^{i} \mathcal{H}_{i}\right\},
\end{aligned}
$$

for $\mu=0,1, \ldots, 4 k+2$, and

$$
\begin{aligned}
\mathcal{H}_{\perp} & =\frac{1}{2}\left[\mathbb{P}^{2}+2 T\left|B^{2}\right|+T^{2} \operatorname{det} h\right], \\
\mathcal{H}_{i} & =\partial_{i} X^{\mu} P_{\mu}+(B \times B)_{i},
\end{aligned}
$$

where $h$ is the induced space metric $\left(h_{i j}=\partial_{i} X^{\mu} \partial_{j} X^{\nu} \eta_{\mu \nu}\right.$ for $i, j=1, \ldots, 4 k+1)$. Also,

$$
\mathbb{P}_{\mu}=P_{\mu}-T C_{\mu},
$$

where, for $d=4 k+1$,

$$
C_{\mu}=\frac{1}{d !} \varepsilon_{\mu \nu_{1} \ldots \nu_{d}} \varepsilon^{i_{1} \ldots i_{d}} \partial_{i_{1}} X^{\nu_{1}} \cdots \partial_{i_{d}} X^{\nu_{d}} .
$$

The replacement $P_{\mu} \rightarrow \mathbb{P}_{\mu}$ serves the same purpose as before: subtraction of $T$ from the Monge-gauge energy density, as can be verified using the identities $\partial_{i} X^{\mu} C_{\mu} \equiv 0$ and $C^{2} \equiv-\operatorname{det} h$.

The Monge gauge choice $\left(X^{0}, \mathbf{X}\right)=(t, \boldsymbol{\sigma})$ takes the covariant action of (39) to the action of (34) with Hamiltonian density (35) for any $k$, but the validity of this step depends on the first-class property of the constraints because they will not otherwise generate the required gauge invariances. Thus, the problem of finding a Hamiltonian 
density $\mathcal{H}$ that ensures a Lorentz invariant action, which has been addressed by a very different method in [14], is here transferred to the problem of finding first-class constraint functions for the covariant action. It is again convenient to choose a functional basis for them by defining

$$
H_{\perp}[\beta]=\int d \boldsymbol{\sigma} \beta \mathcal{H}_{\perp}, \quad H_{\|}[\boldsymbol{\alpha}]=\int d \boldsymbol{\sigma} \alpha^{i} \mathcal{H}_{i}
$$

for vector field $\boldsymbol{\alpha}$ and scalar inverse-density $\beta$ on the $(4 k+1)$ space. The relevant PB relations are

$$
\begin{gathered}
\left\{X^{\mu}(\boldsymbol{\sigma}), P_{\nu}(\boldsymbol{\varsigma})\right\}_{P B}=\delta_{\nu}^{\mu} \delta(\boldsymbol{\sigma}-\boldsymbol{\varsigma}), \\
\left\{A_{i_{1} \ldots i_{n}}(\boldsymbol{\sigma}), B^{j_{1} \ldots j_{n}}(\boldsymbol{\varsigma})\right\}_{P B}=\frac{1}{2 n !} \delta_{\left[i_{1}\right.}^{j_{1}} \cdots \delta_{\left.i_{n}\right]}^{j_{n}} \delta(\boldsymbol{\sigma}-\boldsymbol{\varsigma})
\end{gathered}
$$

for $n=2 k$. The second line generalizes to arbitrary $k$ the relation $\left\{\varphi(\sigma), \varphi^{\prime}(\varsigma)\right\}_{P B}=\frac{1}{2} \delta(\sigma-\varsigma)$, which follows directly from the canonical PB relations of (20).

Using these $\mathrm{PB}$ relations we may compute the PBs of the constraint functions (40); for $T=0$ one finds that the only nonzero $\mathrm{PB}$ relations are

$$
\begin{aligned}
& \left\{H_{\|}[\boldsymbol{\alpha}], H_{\perp}[\beta]\right\}_{P B}=H_{\perp}\left[\mathcal{L}_{\boldsymbol{\alpha}} \beta\right], \\
& \left\{H_{\|}[\boldsymbol{\alpha}], H_{\|}[\tilde{\boldsymbol{\alpha}}]\right\}_{P B}=H_{\|} \llbracket \boldsymbol{\alpha}, \tilde{\boldsymbol{\alpha}} \rrbracket,
\end{aligned}
$$

where $\mathcal{L}_{\alpha} \beta$ is the Lie derivative of $\beta$ with respect to the vector field $\alpha$. This is exactly what one finds for the null $(4 k+1)$-brane, so we may impose the Monge gauge to arrive at the action (34) with Hamiltonian density (38). This is the promised class of conformal chiral $2 k$-form electrodynamics that includes, as the $k=0$ case, the FJ chiral boson theory.

For $k=1$, the action (34) is Lorentz invariant for the more general $T$-dependent Hamiltonian density of (35). In this case one finds that

$$
\left\{H_{\perp}[\beta], H_{\perp}[\tilde{\beta}]\right\}_{P B}=H_{\|}[\boldsymbol{\alpha}(\beta, \tilde{\beta})],
$$

where

$$
\alpha^{i}(\beta, \tilde{\beta})=\left[T^{2}(\operatorname{det} h) h^{i j}+2 T\left[B^{2}\right]^{i j}\right]\left(\beta \stackrel{\leftrightarrow}{\partial}_{j} \tilde{\beta}\right),
$$

with $\left[B^{2}\right]^{i j}=B^{i k} B^{j l} h_{k l}$. The right-hand side of (46) apparently includes a quartic term in $B$ that cannot appear on the left-hand side but the identity $\left[B^{2}\right]^{i j}(B \times B)_{j} \equiv 0$ ensures that it is absent [8]. This identity fails for $k>1$, so the Hamiltonian density of (35) does not define a Lorentz invariant theory for $k>1$ unless $T=0$. There may exist a modified choice of $\mathcal{H}$ that overcomes this restriction, but the $k=2$ example provided by IIB supergravity [15] suggests that nonconformal interactions for $k>1$ must be gravitational.

I am grateful to Igor Bandos, Gary Gibbons, and Dmitri Sorokin for bringing to my attention relevant earlier work, and for other helpful comments on an earlier version of this Letter. This work was partially supported by STFC consolidated Grant No. ST/P000681/1.

Note added-Conformal $n$-form electrodynamics in spacetime dimension $2(n+1)$ for any $n$, generalising the $n=1$ case of Bialynicki-Birula [12], has been studied previously by Chruscinski [16], but without the imposition of a chirality constraint for even $n$.

[1] M. Henneaux and C. Teitelboim, P-form electrodynamics, Found. Phys. 16, 593 (1986).

[2] M. Henneaux and C. Teitelboim, Dynamics of chiral (selfdual) $P$-forms, Phys. Lett. B 206, 650 (1988).

[3] N. Marcus and J. H. Schwarz, Field theories that have no manifestly Lorentz invariant formulation, Phys. Lett. 115B, 111 (1982).

[4] P. Pasti, D. P. Sorokin, and M. Tonin, On Lorentz invariant actions for chiral $p$ forms, Phys. Rev. D 55, 6292 (1997); Covariant action for a $D=11$ five-brane with the chiral field, Phys. Lett. B 398, 41 (1997).

[5] A. Sen, Self-dual forms: Action, Hamiltonian and compactification, J. Phys. A 53, 084002 (2020).

[6] R. Floreanini and R. Jackiw, Self-Dual Fields as Charge Density Solitons, Phys. Rev. Lett. 59, 1873 (1987).

[7] G. W. Gibbons and P.C. West, The metric and strong coupling limit of the M5-brane, J. Math. Phys. (N.Y.) 42, 3188 (2001).

[8] P. K. Townsend, An interacting conformal chiral 2-form electrodynamics in six dimensions, arXiv:1911.01161.

[9] A. Schild, Classical null strings, Phys. Rev. D 16, 1722 (1977).

[10] J. Stachel, Thickening the string. II. The null string dust, Phys. Rev. D 21, 2182 (1980).

[11] E. Bergshoeff, D. P. Sorokin, and P. K. Townsend, The M5brane Hamiltonian, Nucl. Phys. B533, 303 (1998).

[12] I. Bialynicki-Birula, Nonlinear electrodynamics: Variations on a theme by Born and Infeld, in Quantum Theory of Particles and Fields: Birthday Volume Dedicated to Jan Łopuszański, edited by B. Jancewicz and J. Lukierski (World Scientific, Singapore, 1983), pp 31-48; Field theory of photon dust, Acta Phys. Pol. B 23, 553 (1992).

[13] L. Mezincescu and P. K. Townsend, DBI in the IR, J. Phys. A 53, 044002 (2020).

[14] G. Buratti, K. Lechner, and L. Melotti, Self-interacting chiral $p$-forms in higher dimensions, Phys. Lett. B 798, 135018 (2019).

[15] J. H. Schwarz and P. C. West, Symmetries and transformations of chiral $N=2 D=10$ supergravity, Phys. Lett. 126B, 301 (1983).

[16] D. Chruscinski, Strong field limit of the Born-Infeld p-form electrodynamics, Phys. Rev. D 62, 105007 (2000). 